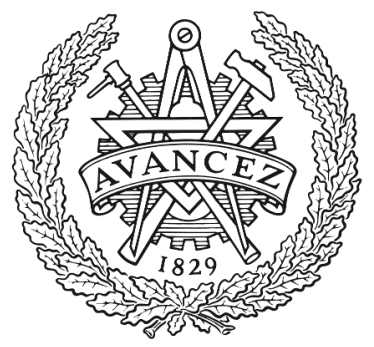

CHALMERS

UNIVERSITY OF TECHNOLOGY

\title{
Understanding Interactions Driving the Template-Directed Self-Assembly of Colloidal Nanoparticles at Surfaces
}

Downloaded from: https://research.chalmers.se, 2023-04-26 07:04 UTC

Citation for the original published paper (version of record):

Eklöf, J., Löfgren, J., Erhart, P. et al (2020). Understanding Interactions Driving the

Template-Directed Self-Assembly of Colloidal

Nanoparticles at Surfaces. Journal of Physical Chemistry C, 124(8): 4660-4667.

http://dx.doi.org/10.1021/acs.jpcc.0c00710

N.B. When citing this work, cite the original published paper. 


\title{
Understanding Interactions Driving the Template-Directed Self- Assembly of Colloidal Nanoparticles at Surfaces
}

\author{
Johnas Eklöf-Österberg, Joakim Löfgren, Paul Erhart,* and Kasper Moth-Poulsen*
}

Cite This: J. Phys. Chem. C 2020, 124, 4660-4667

Read Online

\section{ACCESS |}

Wlll Metrics \& More

Article Recommendations

Supporting Information

\begin{abstract}
Controlled deposition of colloidal nanoparticles using self-assembly is a promising technique for, for example, manufacturing of miniaturized electronics, and it bridges the gap between top-down and bottom-up methods. However, selecting materials and geometry of the target surface for optimal deposition results presents a significant challenge. Here, we describe a predictive framework based on the Derjaguin-LandauVerwey-Overbeek theory that allows rational design of colloidal nanoparticle deposition setups. The framework is demonstrated for a model system consisting of gold nanoparticles stabilized by trisodium citrate that are directed toward prefabricated sub-100 nm features on a silicon substrate. Experimental results for the model system are presented in conjunction with theoretical analysis to assess its reliability. It is shown that threedimensional, nickel-coated structures are well suited for attracting gold nanoparticles and that optimization of the feature geometry based on the proposed framework leads to a systematic improvement in the number of successfully deposited particles.
\end{abstract}

\section{INTRODUCTION}

Guided assembly of nanosized particles (NPs) and clusters ${ }^{1}$ onto surfaces is of interest for the continued miniaturization of circuits using molecular electronics and other applications, for example, in plasmonic-based sensors, ${ }^{2-4}$ stimulation of cell adhesion at nanostructured interfaces ${ }^{5}$ and the engineering of neuronal cell function. ${ }^{6}$

Early investigations revealed that a Coulomb blockade ${ }^{7,8}$ can be measured through a quantum dot or an NP when positioned between two electrodes. Bar-Joseph et al. subsequently demonstrated that a circuit could be constructed with NP dimers interlinked by molecules and electrostatically captured between electrodes. 9,10 This method, however, only allows for the capture of a single NP or a dimerized NP pair. In order to compete with conventional semiconductor integrated circuits, methods capable of simultaneously constructing nanofeatures and nanogaps in parallel ${ }^{11,12}$ and assembling multiple nanoparticles onto specific sites on a sample or device are required. ${ }^{13}$

Previously, methods for parallel NP assembly have been proposed based on, for example, meniscus flow, ${ }^{14-16}$ direct NP growth onto the sites of interest, ${ }^{17}$ selective chemical activation and passivization of a surface, ${ }^{18,19}$ or polymer-templated selfassembly. ${ }^{20,21}$ Recently, a facile method for parallel delivery of NPs to selected parts of a sample was put forward by Eklöf et al. $^{22}$ based on wet chemical deposition. The key idea is to leverage colloidal interactions between suspended nanoparticles and prefabricated nanosized features (nanofeatures) on a substrate to guide particles toward target areas on the sample (Figure 1). The technique has been demonstrated for both single and dimerized gold nanoparticles stabilized by

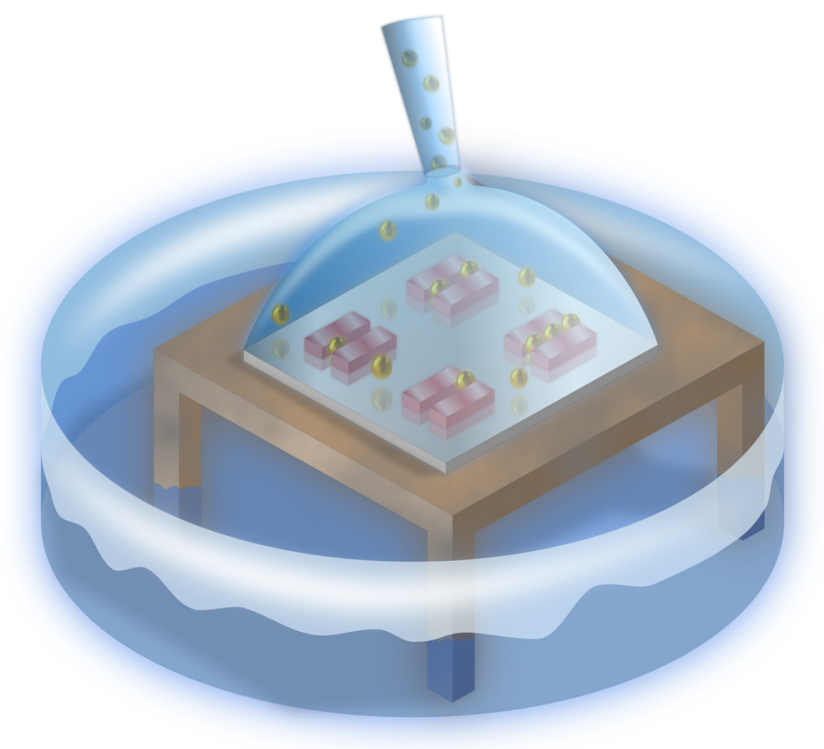

Figure 1. Schematic of the depositing setup. A chip with a variety of prefabricated structures is positioned on a three-dimensional printed scaffold placed in a beaker of water. A drop containing the NP dispersion is placed on top of the chip, and everything is sealed with a glass lid in order to prevent evaporation of the drop.

Received: January 26, 2020

Published: February 4, 2020 
trisodium citrate using patches of metal coating and threedimensional structures. ${ }^{23,24}$

While colloidal deposition has many advantages for guided nanoparticle assembly, including good scalability and no need for chemical pretreatments of the surface, it also presents many challenges. In particular, the combination of a complex chemical environment with the stochastic nature of deposition events renders an uncertainty regarding the positioning of NPs. To remedy this situation, we here present a framework for rational design of guided NP deposition based on the Derjaguin-Landau-Verwey-Overbeek (DLVO) theory. As a model system, we consider the deposition of citrate-stabilized AuNPs on metal-coated nanofeatures due to its key role in the development of the experimental methodology. Particular emphasis is put on design aspects such as the choice of the metal nanofeatures and the selection of optimal shapes. To keep the model system realistic for applications in small-scale electronics and to restrict the design space, the nanofeatures considered generally consist of two identical parts placed in a mirrored configuration to represent electrodes. Complementary to the modeling, experimental depositions have been carried out for the model system in order to gauge the predictive power of the theoretical analysis.

Two common simplifications found in the literature regarding the DLVO theory are avoided in the implementation of our framework. First, instead of the Derjaguin approximation, ${ }^{25,26}$ the surface element integration (SEI) method ${ }^{27,28}$ is employed. This enables the description of particle-surface interactions in the presence of both chemical ${ }^{29-31}$ and spatial heterogeneities ${ }^{32-34}$ and has the additional advantage of being applicable even when the characteristic range of the interactions is on the order of the diameter of the particle. Second, we do not linearize the Poisson-Boltzmann (PB) equation, and the solution of which is required when calculating the electrostatic double layer (EDL) contribution to the interaction energy. This is motivated in part by the fact that the experimental electrolyte consists mainly of dissolved citrate, an asymmetric 3:1 salt. From a broader perspective, however, linearization of the $\mathrm{PB}$ equation is not valid when the surface potential is on the order of the average thermal energy, which is frequently the case in the type of deposition setups of interest here. ${ }^{24}$

\section{EXPERIMENTAL SECTION}

Citrate-stabilized NPs (Sigma-Aldrich, 742015) with a diameter of $60 \mathrm{~nm}$ were used in the following experiments. Nanofeatures were fabricated using a double-layer resist system consisting of a $100 \mathrm{~nm}$-thick lift of resist (MCC NANO Copolymer EL6, Microlithography Chemicals Corp.) and a 50 nm-thick e-beam resist (6200.13:Anisol 1:2; supplier, Allresist $\mathrm{GmBH})$, all spin-coated onto a $\mathrm{Si}(100)$ wafer with a $400 \mathrm{~nm}$ thick thermally grown $\mathrm{SiO}_{2}$ layer. The wafer was exposed to an electron beam lithography system (JEOL JBX-9300FS, operating at $100 \mathrm{kV}$ ) according to the predesigned pattern described below. A $70 \mathrm{~nm}$-thick metal layer of $\mathrm{Ni}$ was evaporated after development followed by a lift-off in acetone. The NP concentration in the system was increased over two centrifugation cycles $(2400 \mathrm{~g}, 10 \mathrm{~min})$, where excess solution was replaced with deionized water after each cycle.

2-Propanol was then mixed into the particle dispersion. A droplet of the dispersion was placed on a chip supported by a homebuilt setup with controlled humidity in order to reduce evaporation of the droplet. ${ }^{2,24}$ The droplet was rinsed away after the deposition with a mixture of 2-propanol and deionized water (in the same ratio as the dispersion), and the chip was rinsed with deionized water and blow-dried under a stream of $\mathrm{N}_{2}$. The schematic of the setup is shown in Figure 1. A preliminary investigation of the samples was carried out with an optical dark-field microscope (Carl Zeiss Microscopy $\mathrm{GmbH}$ : Axio) where larger parts of the sample were inspected for deposited particles. A more thorough scanning electron microscopy (SEM) investigation was then performed at $7 \times$ $10^{-7}$ mbar using the $30 \mu \mathrm{m}$ aperture of a Zeiss Supra $60 \mathrm{VP}$ equipped with an in-lens detector with an accelerating voltage of $12 \mathrm{kV}$.

\section{DEPOSITION ENERGETICS}

Due to the characteristic length and timescales of the systems involved, atomistic treatment of colloidal deposition problems is prohibitively expensive. Hence, studies are typically conducted on a continuum level within the framework of the DLVO theory, ${ }^{35}$ where the interaction free energy between two objects is taken as the sum of a van der Waals (vdW) contribution and a contribution from electrical double layer (EDL) interactions.

The key quantity that must be obtained is the interaction free energy between a depositing particle and a flat surface. This is accomplished using the SEI approach, which has shown to yield vdW energies that match the exact analytic solution for sphere-surface interactions and EDL energies derived from finite element solutions of the PB equation. ${ }^{27}$ In SEI, the interaction energy $U_{f}$ is calculated as the sum of contributions coming from area elements of the particle surface interacting with a flat surface $f$. In particular, for spherical particles, $U_{f}$ only depends on the distance of the closest approach, $D$, between the particle and flat surface. The vdW contribution to $U_{f}$ can be evaluated analytically, ${ }^{36}$ but the EDL contribution requires a numerical solution of the nonlinear $\mathrm{PB}$ equation ${ }^{37}$ due to the presence of citrate, an asymmetric 3:1 salt. A more detailed account of the relevant parts of the DLVO theory and SEI and how to efficiently evaluate $U_{f}$ can be found in the Supporting Information.

Once $U_{f}$ is known as a function of $D$, we can determine if a surface is amenable to deposition of particles. For an NP with potential $\psi_{\mathrm{p}}$ interacting with a flat surface with potential $\psi_{f}$, a repulsive $E D L$ interaction will lead to a free-energy barrier $E_{\mathrm{B}}$ that the particle has to overcome before it can attach to the surface. This barrier arises from the competition between the EDL interaction and the attractive $\mathrm{vdW}$ interaction (Figure S1). Steric interactions arising due to the stabilized layer of citrate formed around the NPs are neglected in this work. Their contribution to the free energy can in principle be evaluated, ${ }^{38,39}$ given sufficient knowledge of the structure and properties of the ligand layer, ${ }^{40-42}$ and affect the location and depth of the free-energy minima corresponding to deposition. Here, we adopt a pragmatic modeling approach where we are primarily concerned with determining if NPs will deposit at all, as determined by the free-energy barrier. For realistic parameter choices, this barrier occurs beyond the extent of the citrate layer (Figure S2) and hence a short-ranged repulsive force is not included.

In general, deposition of NPs onto a surface $f$ is probable if the energy barrier $E_{\mathrm{B}}$ is on the order of $k_{\mathrm{B}} T$ or smaller. In this context, we adopt the notation $\Psi_{f}^{\circ}\left[E_{\mathrm{B}}\right]$ for the surface potential required to yield a barrier of height $E_{\mathrm{B}}$ and refer to this as the deposition potential corresponding to $E_{\mathrm{B}}$. As we shall see, the 
deposition potential is a useful quantity when selecting materials for a deposition setup.

The interaction of a depositing particle with a nanofeature can be obtained by regarding the surrounding walls of the feature as a set of flat surfaces and summing up the contributions $U_{f}$ coming from each wall:

$$
U(r)=\sum_{f} U_{f}(D(r))
$$

Here, $r$ is the position of the particle center of mass, and $D(r)$ is the distance of the closest approach from $r$ to the surface $f$. Since the nanofeatures considered in this work consist of two identical parts placed in a two-fold axis configuration, they enclose a region that we refer as the nanogap. The nanogap volume is bound by the inner walls of the nanofeature and the plane defined by its top surface. A scalar measure of the attractiveness of a general nanogap can then be obtained by integrating $U(r)$ over $V V$, the partial nanogap volume that is accessible to the particle

$$
I[U]=\int_{V} U(r) \mathrm{d} V
$$

While $I[U]$ clearly cannot encode the full complexity underlying a deposition event, it possesses several key characteristics expected of an ideal measure. These include scaling with the volume of the nanogap and the fact that a more negative $I[U]$ indicates a larger chance of deposition occurring and vice versa.

As a complement to the theoretical description of the model, we have made the source code available as a Python package ${ }^{1}$.

\section{SELECTION OF MATERIALS}

We first discuss the importance of the choice of materials in achieving selective deposition of NPs, with an emphasis on the underlying interactions and the guidance offered by the DLVO theory.

As a consequence of the negative charges present on both the citrate-stabilized AuNPs and an unmodified $\mathrm{SiO}_{2}$ substrate and the relatively low Hamaker constant (eq S3) of the latter, the energy barrier for deposition is effectively insurmountable. ${ }^{22}$ Targeted deposition on $\mathrm{SiO}_{2}$ substrate can then be achieved by introducing patches or structures coated with a material that attracts NPs. ${ }^{23,24}$ For this purpose, it is natural to consider metals as candidate materials as they generally have higher Hamaker constants than non-metals; ${ }^{43}$ hence, the attractive vdW force between a transition-metal surface and an NP is correspondingly stronger.

The number of candidate materials can be narrowed down by calculating the theoretical deposition potential of the particle-surface system under different conditions. To illustrate the process, we analyzed the deposition potential $\Psi_{f}^{\circ}\left[k_{\mathrm{B}} T\right]$ as a function of the particle surface potential for our citrate-stabilized AuNP model system (Figure 2). The use of a target energy barrier $E_{\mathrm{B}}=k_{\mathrm{B}} T$ in these calculations implies that the calculated deposition potentials correspond to a system where NP deposition occurs rapidly since a large fraction of particles will have a kinetic energy in the range of the barrier. Other values for the target barrier are possible; for instance, to study the limit where deposition becomes improbable, one can consider a larger value, for example, $\Psi_{f}^{\circ}\left[10 k_{\mathrm{B}} T\right]$. Two surfaces were included in the analysis with Hamaker constants $A_{\mathrm{H}}\left[\mathrm{SiO}_{2}\right]=7.2 \times 10^{-20} \mathrm{~J}^{44}$ and $A_{\mathrm{H}}[\mathrm{M}]=40 \times 10^{-20} \mathrm{~J}$. The a)

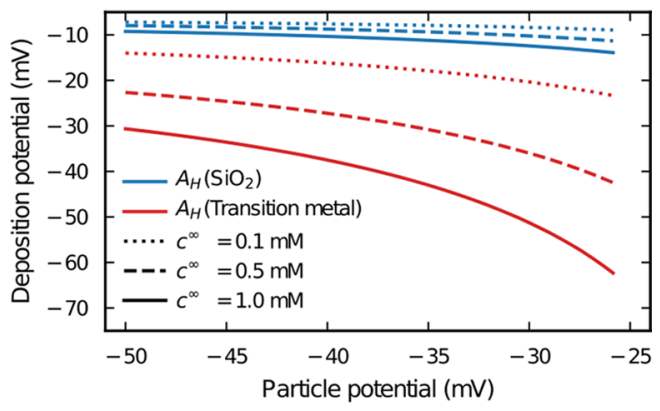

b)

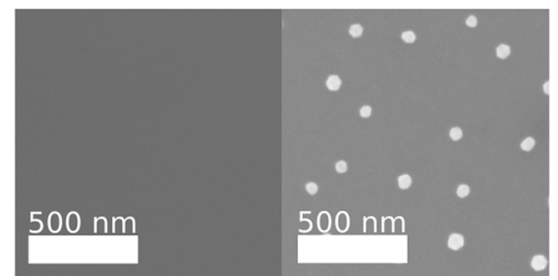

Figure 2. (a) Material selection for targeted NP deposition can be simplified by a deposition potential analysis. Particle potentials are mapped onto the surface potential that a candidate material with a fixed Hamaker constant would need in order to achieve a given deposition energy barrier $E_{\mathrm{B}}$. Illustrated here is the $E_{\mathrm{B}}=k_{\mathrm{B}} T$ case, corresponding to rapid NP deposition. (b) SEM micrographs of trisodium citrate-stabilized NPs after deposition on $\mathrm{SiO}_{2}$ at the top and $\mathrm{NiO}$ at the bottom. The arrows point toward corresponding plots in (a).

latter value is typical for the Hamaker constant of a late transition metal M. ${ }^{43}$ Given $A_{\mathrm{H}}[\mathrm{Au}]=45 \times 10^{-20} \mathrm{~J}$ and $A_{\mathrm{H}}\left[\mathrm{H}_{2} \mathrm{O}\right]=4.8 \times 10^{-20} \mathrm{~J},{ }^{43,45}$ effective Hamaker constants for all vdW interactions in the system could subsequently be determined using the standard combining rule (eq S4). Another important consideration is the citrate concentration for which we note that the centrifugation and partial replacement of solvent with deionized water leads to an estimated range on the order of $0.1-1 \mathrm{mM}$, that is, lower than what is typically used for synthesis of citrate-stabilized AuNPs.

If the measured $\zeta$ potential of $-34 \mathrm{mV}^{22}$ of the AuNPs is taken as an approximation of the AuNP potential, we can conclude, on the basis of Figure 2a, that rapid NP deposition can be achieved on a metal surface even if the surface potential is negative (Figure 2a). In this case, the EDL interaction is still repulsive but deposition can occur since the $\mathrm{vdW}$ interaction is strong enough to overcome it over distances on the order of the system's Debye length (eq S9). At $c^{\infty}=1 \mathrm{mM}$ citrate concentration, a metal surface potential of $-40 \mathrm{mV}$ would, in fact, still yield a barrier height of $E_{\mathrm{B}}=k_{\mathrm{B}} T$. For a surface with a smaller Hamaker constant than $\mathrm{SiO}_{2}$, rapid NP deposition is still possible for negative surface potentials not exceeding approximately $-10 \mathrm{mV}$ in magnitude. In this case, however, concentration dependence is less pronounced. These results thus underscore the sensitivity of the outcome of a deposition experiment to the Hamaker constants of the chosen materials.

The usefulness of a theoretical analysis in terms of deposition potentials depends upon whether the surface potentials of the candidate materials can be estimated. In ref $24, \mathrm{Ni}$ was found to be highly amenable to deposition of citrate-stabilized AuNPs dispersed in $1 \mathrm{mM} \mathrm{KCl}^{24}$ in which case the measured surface potential was $\psi_{f}=61.9 \mathrm{mV}$. Since 
$A_{\mathrm{H}}[\mathrm{Ni}] \approx 45 \times 10^{-20} \mathrm{~J},{ }^{43}$ this is consistent with Figure 2. Indeed, for positive surface potentials, the EDL interaction with citrate-stabilized AuNP is attractive; hence, in isolation from other surfaces and NPs, there can be no barrier toward deposition. The same study also found that no deposition took place on $\mathrm{SiO}_{2}$, for which a surface potential $\psi_{f}=-57.3 \mathrm{mV}$ was measured. This is again consistent with Figure $2 \mathrm{a}$, as $-57.3 \mathrm{mV}$ is a significantly more negative potential than the $-10 \mathrm{mV}$ that would be required to obtain a barrier height of $E_{\mathrm{B}}=k_{\mathrm{B}} T$.

Deposition of AuNPs on $\mathrm{Ni}$ and $\mathrm{SiO}_{2}$ surfaces carried out as part of the present study confirms this picture: AuNPs are only found on the Ni surface (Figure $2 b$ ). No new surface potential measurements have been made in this case, however, due to the significant effort and difficulty they entail. Since surface potentials are not transferable when different electrolytes are considered, we can, however, only expect to gain qualitative understanding from the DLVO theory in the present case.

Fully realizing the potential of the DLVO theory as a tool for analyzing and predicting the outcome of deposition problems would require a multiscale approach where atomistic methods are used to calculate the prerequisite surface potentials. ${ }^{46,47}$

\section{GEOMETRY OPTIMIZATION}

In addition to the choice of NPs and sample materials, the impact of the nanofeature geometry on the outcome of the deposition process was investigated. The primary design goal, according to which different nanofeatures were selected for evaluation, was their conduciveness to NPs depositing inside the nanogap. Theoretically, we quantify the attractiveness of a nanofeature using the integrated free energy, $I[U]$, introduced in eq 2. Changes in $I[U]$ are then studied as a function of the design parameters of selected nanofeatures. Typically, the final application of the deposited nanoparticles imposes some restrictions on the maximum allowed area for the nanogaps, which is taken into account when comparing different designs.

The simplest nanofeature design considered consists of two metal bars positioned in parallel to partially enclose a nanogap. If the dimension of each bar is fixed, the only adjustable design parameter of this feature is the spacing between the bars, that is, the nanogap width. Experimentally, deposition of citratestabilized AuNPs was observed on parallel Ni bar features for a range of nanogap widths. As apparent from SEM micrographs (Figure 3a,b), deposition of NPs occurs both inside the nanogap and on the outside walls of the Ni bars. Proximity to a single surface of $\mathrm{Ni}$ is thus enough to overcome the repulsion from the $\mathrm{Si}$ substrate, consistent with the assignment of a positive surface potential to the $\mathrm{Ni}$ surface, as discussed in the previous section. A careful examination of the SEM images reveals that no particles are found in the middle of the nanogaps, indicating that the decay of the EDL interactions over the length of the nanogap is rapid enough for a deposition close to one of the $\mathrm{Ni}$ surface to be more favorable than deposition in between the two surfaces. The parallel bar geometry is thus not optimal for deposition since a particle cannot attain sufficient proximity to more than one of the nanofeature walls. Furthermore, since increasing the nanogap width from 50 to $125 \mathrm{~nm}$ results in a slight decrease in the number of successfully deposited particles (Figure 3c), the nanogap width as a design parameter does not offer much control over the deposition results. Further insight into the parallel bar geometry can be obtained from the DLVO theory. For this purpose, the interaction free energy between a AuNP and the surrounding sample was calculated over a vertical a)

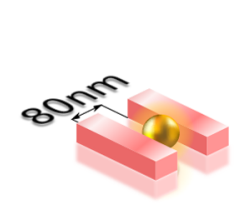

b)
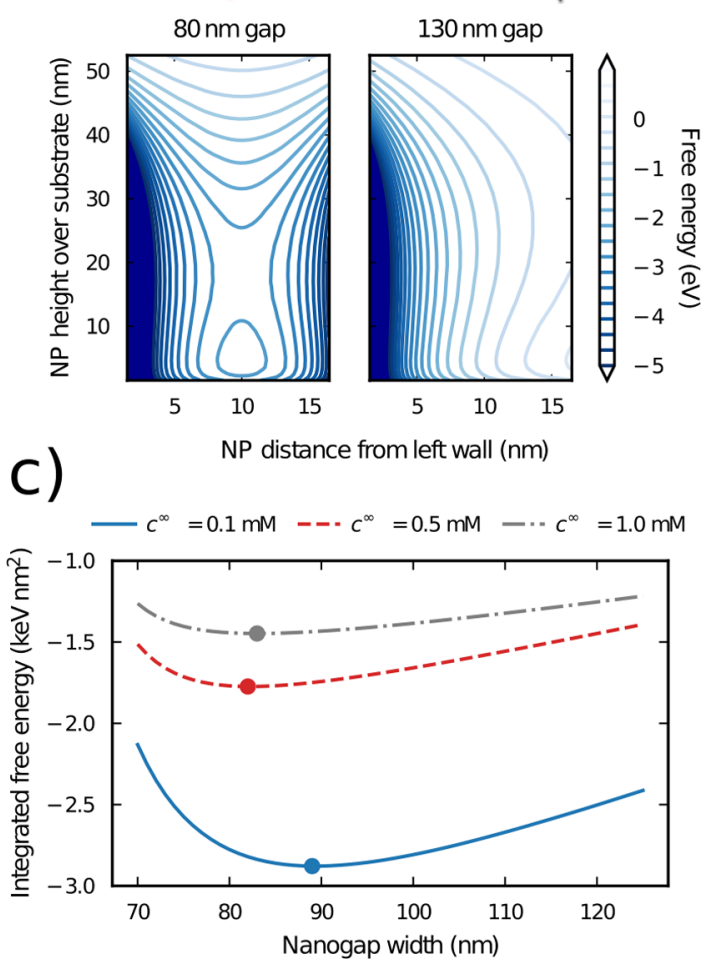

Figure 3. Cross-sectional attraction maps for two parallel Ni bars with a spacing of (a) 85 and (b) $125 \mathrm{~nm}$. The $x$ axis indicates the distance from an NP to the wall of one bar. Only the left wall is shown in the map. Each map has a corresponding schematic above; the cross section of which can be seen in (b). (c) Integrating the free energy throughout planar cuts made perpendicular to the parallel $\mathrm{Ni}$ bar geometry gives a measure of the overall ability of the nanogap to attract nanoparticles. This measure attains a maximum when the nanogap is slightly wider than the particle diameter, although the effect is less pronounced at higher salt concentrations.

plane spanning the nanogap between the bars (Figure 3a, top). In these calculations, the surface potentials were set to representative values $\psi_{f}=-\psi_{\mathrm{p}}=-\psi_{\mathrm{s}}=50 \mathrm{mV}$ and a citrate concentration of $1.0 \mathrm{mM}$ was assumed. From the resulting free-energy maps, it can be seen that the most favorable positions for the particle are, as expected from the experimental results, those closest to a Ni surface (Figure 3a, middle) for both narrow $80 \mathrm{~nm}$ nanogaps and wider $125 \mathrm{~nm}$ gaps.

In addition to the free-energy maps, the integrated free energy was obtained for the parallel bar geometry as a function of the nanogap width under different concentrations (Figure $3 c){ }^{2}$ Intuitively, one might expect an increase in $I[U]$ with nanogap width since a wider gap exposes more of the repulsive substrate, which can also be seen by comparing the energy maps for the narrow and wide gaps (Figure 3a,b). Starting from narrow $70 \mathrm{~nm}$ gaps, however, $I[U]$ initially becomes more negative and the expected increase is only exhibited for gaps wider than $80 \mathrm{~nm}$, depending on the concentration. As a result, a minimum exists corresponding to the optimal nanogap width most likely to capture an NP. This minimum occurs at $98 \mathrm{~nm}$ when $c^{\infty}=1 \mathrm{mM}$ and at around $82 \mathrm{~nm}$ for 
concentrations between $0.5-1.0 \mathrm{mM}$. The overall trend is that, for lower concentrations $c^{\infty} \ll 1 \mathrm{mM}$, the minima are both deeper and more pronounced, indicating that the nanogap is more attractive but its capture efficiency is also more sensitive to concentration.

While the parallel bar design is good at capturing particles, it does not allow lateral confinement of the deposited particles, which becomes a problem when high-precision positioning is necessary. It is possible to simultaneously remedy this issue and improve the probability for successful particle deposition by considering geometries where the nanogap is enclosed by $\mathrm{Ni}$ walls to a larger extent. For this purpose, geometries where the two nanofeature parts resemble opposing forklifts were constructed. If the maximum nanogap area is restricted, geometries belonging to the forklift family can achieve more negative $I[U]$ values than parallel bar geometries since, in the simplest case, a rectangular forklift geometry can be constructed by extending the parallel bar setup with walls that partially enclose those sides of the nanogap previously left uncovered (Figure 4a,b). Further improvement of the

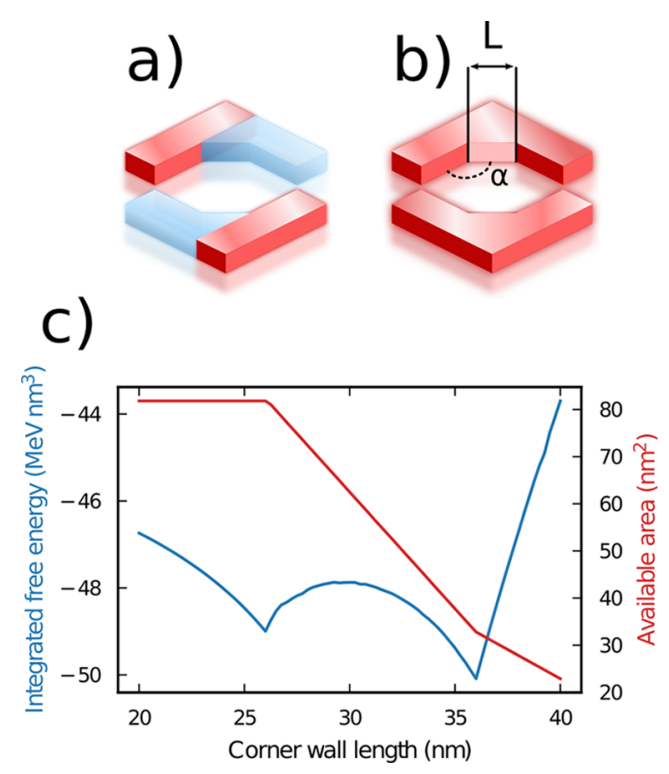

Figure 4. Schematic representation of successive improvements to a parallel bar design. (a) Attractive walls are added to the sides to better enclose the target deposition region. (b) Sharp inner corners are cut away and replaced by corner walls with an optimal opening angle of $135^{\circ}$. Finally, the length of the corner wall can be tuned. If the opening angle remains fixed, extending the length of the corner wall beyond a certain point leads to a decrease in the nanogap area available for particle deposition; hence, the optimal choice of the corner length is not immediately clear.

rectangular forklift design can be achieved by cutting away the $90^{\circ}$ corners at an angle $\alpha$ and introducing a new corner wall along the direction of the cut (Figure $4 b, c$ ). Symmetry dictates that the optimal opening angle $\alpha$ of the corner wall must be close to $135^{\circ}$, which is also confirmed by calculations (Figure S3).

The remaining design parameter to optimize is the length of the corner wall, denoted as $L$. From Figure $4 b$, it can be seen that the integrated free energy exhibits a nontrivial dependence on this length, with two distinct minima at 26 and 36, the latter being the global minimum. The existence of these two minima closely reflects changes in the nanogap area available to a depositing particle. More precisely, the first minima occurs when the corner wall reaches its maximally allowed value under the constraint that the available area does not decrease. Further increase in $L$ initially leads to an increase in $I[U]$ due to the associated decrease in available area, but this trend is reversed once regions that were previously far removed from the corner come within the range of its attractive interaction. A second minimum consequently appears when the corner wall has become sufficiently long such that the regions closest to the side walls become unavailable if $L$ is increased further. Contrary to what might be expected, the attractive power of a nanogap can thus, in certain situations, be increased to an extent by reducing the area available for depositing particles in favor of greater proximity to attractive feature surfaces.

Experimentally, a set of four nanofeatures designed for both lateral and vertical confinement, that is, two-dimensional control of depositing NPs, was considered, including the forklift design (Figure 5a-d). Counting the number of
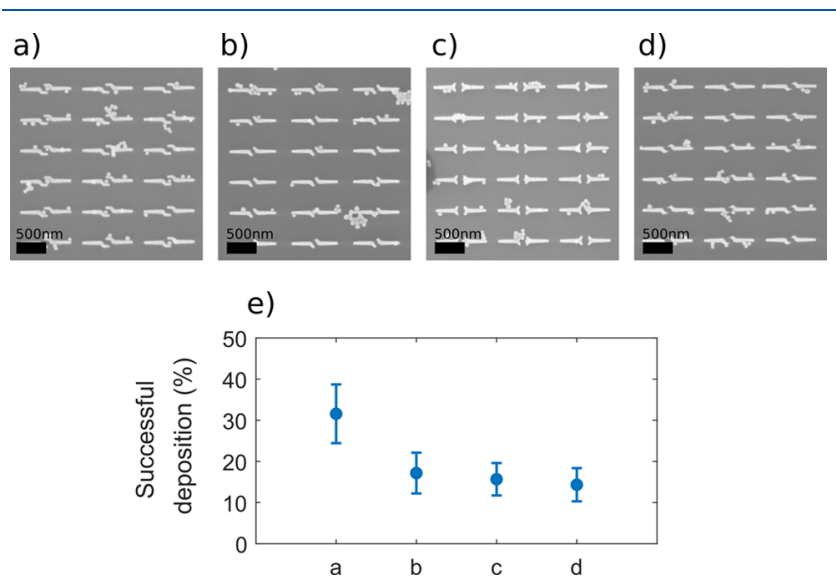

Figure 5. $(a-d)$ Partial SEM micrograph over four different arrays of $70 \mathrm{~nm}$-thick $\mathrm{Ni}$ features. Each array corresponds to a different candidate geometry that offers both lateral and vertical control of the position of depositing NPs. (e) Deposition success rates after a sample submersion period of $72 \mathrm{~h}$.

successful NP depositions on each array yielded a success rate of $31 \%$ for the forklift geometry and lower rates ranging from $14-18 \%$ for the other candidates (Figure 6). In terms of our theoretical model, this result can be attributed to the fact that the forklift nanogap is enclosed to a higher degree by the attractive $\mathrm{Ni}$ walls than the other geometries, which decreases the integrated free energy as seen in Figure 4 and Figure S3.

The forklift geometry was also compared to the parallel bar geometries with varying nanogap widths (Figure 5). Here, the percentage of successfully deposited single NPs is $\sim 40 \%$ for a $50 \mathrm{~nm}$-wide gap and $37 \%$ for a $60 \mathrm{~nm}$-wide gap. The latter number is suitable for comparison with the forklift geometry where the vertical dimension of the nanogap is also $60 \mathrm{~nm}$. This leads to the conclusion that the double bar geometry has a slightly higher success rate for single NP deposits, which is explained theoretically by the increase in available area close to attractive $\mathrm{Ni}$ walls. It must be stressed, however, that the increase in success rate of deposited NPs in this case comes with a complete loss of lateral control over the NPs positioning, rendering the double bar geometry unsuitable for many applications.

Comparisons between different nanofeature geometries are thus most meaningful when the total nanogap area is 
a)

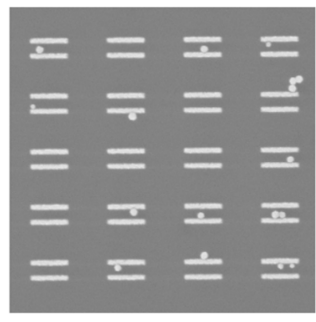

b)

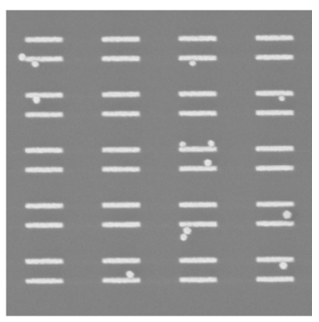

c)

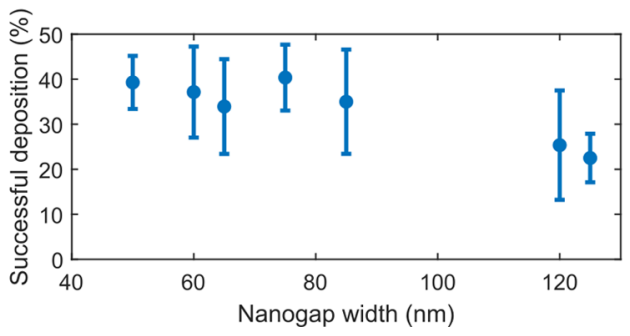

Figure 6. SEM micrographs over two arrays of $\mathrm{Ni}$ bars with a height of $70 \mathrm{~nm}$ on a $\mathrm{Si} / \mathrm{SiO}_{2}$ substrate. The arrays seen in this figure are parts of a greater set of arrays. The parallel bars are spaced with (a) 85 $\mathrm{nm}$ and (b) $125 \mathrm{~nm}$. (c) Percentage of successful depositions of a single NP. NPs were allowed to deposit over a period of $72 \mathrm{~h}$. The full SEM micrographs used in this analysis can be found in the Supporting Information, Figures S4-S10.

constrained from above by the final application, in which case design parameter optimizations of the kind illustrated in Figures 3c,4 and Figure S3 are straightforward to apply.

As a caveat to the theoretical predictions presented above, it should be noted that the optimal ranges of certain design parameters are always wider in experimental settings since particle sizes are not fixed, rather they follow a distribution with a given mean.

\section{CHEMICAL ENVIRONMENT}

In addition to the choice of sample materials and nanofeature geometry, several aspects of the chemical deposition environment merit further discussion.

It was observed that the addition of 2-propanol to the citrate-covered AuNP solution is required to successfully achieve NP deposition. From Figure S11, it can be seen that 2propanol greatly improves the number of NPs successfully deposited on the prefabricated nanostructures. Optimal results were obtained with a $50 \%$ mixture between AuNP solution and 2-propanol, and further addition results in significant NP agglomeration. Intuitively, this could be attributed to the decrease in the Debye length caused by the presence of 2propanol, which implies greater screening of the repulsive electrostatic interactions from the $\mathrm{SiO}_{2}$ substrate. The situation is complicated, however, by the fact that the addition of propanol to the solution can also affect the local chemical environment of the surfaces of $\mathrm{Ni}$ and $\mathrm{SiO}_{2}$ and the AuNPs. ${ }^{48-50}$ A more thorough investigation of the impact of 2-propanol on NP deposition is therefore an important topic for future work. As a consequence, the DLVO-based modeling was restricted to the case of pure water to maintain a consistent set of simulation parameters. This, in turn, implies that our current calculations do not capture the full chemical complexity of the experiments. We emphasize, however, that in spite of this deficiency, the important general trends of the system are captured, as evident from the comparison with experimental data. Furthermore, given a suitable set of parameters for samples where the solvent is a water-propanol mixture, the theoretical analysis can be repeated in much the same fashion.

Another important consideration in designing deposition setups is the role of $\mathrm{pH}$, which influences, for example, the surface potentials and speciation of citrate. In the present work, measurements performed after dilution with propanol yielded a $\mathrm{pH}$ value of 6.9. Under such conditions, the majority of citrate ions in the solution are trivalent, ${ }^{51}$ and hence no other valencies were considered in the DLVO model.

Rinsing and drying of the sample after the liquid-phase deposition is also a point of concern as it can lead to removal of NPs, in particular, if they are weakly bound to the surface. ${ }^{52}$ To investigate whether any significant removal of particles was occurring on our samples, chips were subjected to repeated rinsing and drying cycles with intermediate SEM imaging. The typical result can be seen in Figure S12 where no observable change in the number of deposited NPs is found when comparing the same area of a single chip that has undergone one and three cleaning cycles, respectively.

\section{CONCLUSIONS}

We have presented a predictive framework for analyzing guided deposition of colloidal nanoparticles based on a combination of the DLVO theory and empirical experiments. The framework was illustrated for a system consisting of citrate-stabilized NPs in aqueous solution above sub-100 nm metal/metal oxide nanosized features on a $\mathrm{Si} / \mathrm{SiO}_{2}$ substrate.

With regard to materials, it was found that $\mathrm{Ni}$ is particularly suitable for the deposition of AuNPs, which can be attributed to its surface charge being of opposite sign of the NPs and its high Hamaker constant. We further explored, using our model system of citrate-stabilized AuNPs, how the concept of deposition potentials defined with respect to a fixed value deposition barrier can aid in the process of selecting materials for a deposition setup. In particular, this analysis revealed that the range of surface potentials over which a material supports rapid deposition of NPs is highly sensitive to the Hamaker constant, with larger allowed ranges for metals with high Hamaker constants such as Ni. It was also noted that while the current modeling framework based on DLVO can describe much of the chemical environment, it requires knowledge of parameters that are difficult to measure experimentally such as surface potentials. Here, atomistic simulations could provide a solution for calculating properties that are otherwise difficult to assess.

The selection of optimal geometries for three-dimensional nanofeatures was also investigated. Starting from a basic design consisting of two parallel, bar-shaped nanofeatures, we demonstrated that the design could be systematically improved in terms of the number of successfully deposited particles. More precisely, by extending the bars into angled structures resembling forklifts, the number of successfully deposited nanoparticles could be increased. This type of design also allows a more selective deposition process where the number of nanoparticles captured between opposing structures can be controlled, which is important, for example, for avoiding short circuits in electronics applications.

For more complex nanofeature geometries, additional design parameters are introduced in the form of angles and structure dimensions. Here, the proposed modeling framework offers an inexpensive solution for selecting optimized parameter values 
using the integrated interaction free energy as the objective function, as demonstrated for the forklift geometries.

The model is not only limited toward the deposition of spherical particles; other more complex shapes such as cubes, stars or rods could also be used with some modifications made to the model. One could as well think of more complex structures used to attract the particles, all depending on the application. Only flat surfaces have been considered in this work excluding parameters such as roughness and local defects. Possible continuation for this article could be the deposition inside nanosized trenches or tubes used in, for example, microfluidics.

\section{ASSOCIATED CONTENT}

\section{SI Supporting Information}

The Supporting Information is available free of charge at https://pubs.acs.org/doi/10.1021/acs.jpcc.0c00710.

Extended description of the DLVO theory underlying the modeling framework, interaction energy profiles calculated for NPs close to repulsive surfaces (Figures S1 and S2), implementation details for the modeling framework, design parameter optimization of the forklift opening angle (Figure S3), SEM micrographs of NP deposition on parallel bar nanofeatures with varying nanogap widths (Figures S4-S10), SEM micrographs of NP deposition at different concentrations of 2-propanol (Figure S11), and SEM micrographs of NP deposition on the same sample taken after one and three cycles of rinsing and drying, respectively (Figure S12) (PDF)

\section{AUTHOR INFORMATION}

\section{Corresponding Authors}

Paul Erhart - Department of Physics, Chalmers University of Technology, Gothenburg 41296, Sweden; 이이이.org/00000002-2516-6061; Email: erhart@chalmers.se

Kasper Moth-Poulsen - Department of Chemistry and Chemical Engineering, Chalmers University of Technology, Gothenburg 41296, Sweden; (1) orcid.org/0000-0003-40184927; Email: kasper.moth-poulsen@chalmers.se

\section{Authors}

Johnas Eklöf-Österberg - Department of Chemistry and Chemical Engineering, Chalmers University of Technology, Gothenburg 41296, Sweden

Joakim Löfgren - Department of Physics, Chalmers University of Technology, Gothenburg 41296, Sweden; 자이.org/00000001-6968-5966

Complete contact information is available at:

https://pubs.acs.org/10.1021/acs.jpcc.0c00710

\section{Notes}

The authors declare no competing financial interest.

\section{ACKNOWLEDGMENTS}

This work was supported by the Myfab National Access Program (at MC2 Nanofabrication Laboratory, Chalmers), the Knut and Alice Wallenberg Foundation, Chalmers Area of Advance Nano, and the European Research Council (ERC).

\section{ADDITIONAL NOTES}

${ }^{1}$ https://gitlab.com/joalof/seipy
${ }^{2}$ With the length of the bars kept fixed, the integration required to obtain $I[U]$ can be restricted to a vertical plane.

\section{REFERENCES}

(1) Ni, S.; Wolf, H.; Isa, L. Programmable Assembly of Hybrid Nanoclusters. Langmuir 2018, 34, 2481-2488.

(2) Gschneidtner, T. A.; Fernandez, Y. A. D.; Syrenova, S.; Westerlund, F.; Langhammer, C.; Moth-Poulsen, K. A Versatile Self-Assembly Strategy for the Synthesis of Shape-Selected Colloidal Noble Metal Nanoparticle Heterodimers. Langmuir 2014, 30, 30413050.

(3) Borglin, J.; Guldbrand, S.; Evenbratt, H.; Kirejev, V.; Grönbeck, H.; Ericson, M. B. Insights on proximity effect and multiphoton induced luminescence from gold nanospheres in far field optical microscopy. Appl. Phys. Lett. 2015, 107, 234101.

(4) Lundgren, A.; Hulander, M.; Brorsson, J.; Hermansson, M.; Elwing, H.; Andersson, O.; Liedberg, B.; Berglin, M. GoldNanoparticle-Assisted Self-Assembly of Chemical Gradients with Tunable Sub-50 nm Molecular Domains. Part. Part. Syst. Charact. 2014, 31, 209-218.

(5) Kruss, S.; Wolfram, T.; Martin, R.; Neubauer, S.; Kessler, H.; Spatz, J. P. Stimulation of Cell Adhesion at Nanostructured Teflon Interfaces. Adv. Mater. 2010, 22, 5499-5506.

(6) Gahl, T. J.; Kunze, A. Force-Mediating Magnetic Nanoparticles to Engineer Neuronal Cell Function. Front. Neurosci. 2018, 12, 299.

(7) Averin, D. V.; Likharev, K. K. Coulomb blockade of singleelectron tunneling, and coherent oscillations in small tunnel junctions. J. Low Temp. Phys. 1986, 62, 345-373.

(8) Kastner, M. A. The single-electron transistor. Rev. Mod. Phys. 1992, 64, 849-858.

(9) Dadosh, T.; Gordin, Y.; Krahne, R.; Khivrich, I.; Mahalu, D.; Frydman, V.; Sperling, J.; Yacoby, A.; Bar-Joseph, I. Measurement of the conductance of single conjugated molecules. Nature 2005, 436, $677-680$.

(10) Guttman, A.; Mahalu, D.; Sperling, J.; Cohen-Hoshen, E.; BarJoseph, I. Self-assembly of metallic double-dot single-electron device. Appl. Phys. Lett. 2011, 99, 063113-063111.

(11) Puebla-Hellmann, G.; Mayor, M.; Lörtscher, L. Ultraflat nanopores for wafer-scale molecular-electronic applications. 2015 IEEE 15th International Conference on Nanotechnology (IEEE-NANO); IEEE, 2015.

(12) Wu, Y.; Hong, W.; Akiyama, T.; Gautsch, S.; Kolivoska, V.; Wandlowski, T.; de Rooij, N. F. Batch fabrication of gold-gold nanogaps by E-beam lithography and electrochemical deposition. Nanotechnology 2013, 24, 235302.

(13) Lambrechts, W. Extending Moore's Law through Advanced Semiconductor Design and Processing Techniques; CRC Press, 2018.

(14) Ni, S.; Klein, M. J. K.; Spencer, N. D.; Wolf, H. Cascaded assembly of complex multiparticle patterns. Langmuir 2014, 30, 9095.

(15) Ni, S.; Leemann, J.; Buttinoni, I.; Isa, L.; Wolf, H. Programmable colloidal molecules from sequential capillarity-assisted particle assembly. Sci. Adv. 2016, 2, No. e1501779.

(16) Zhang, J.; Léonard, D.; Mazurczyk, R.; Yeromonahos, C.; Monnier, V.; Géhin, T.; Monfray, S.; Chevolot, Y.; Cloarec, J.-P. Orthogonal chemical functionalization of patterned $\mathrm{Au} / \mathrm{TiW}$ substrate for selective immobilization of nanoparticles. Nanotechnology 2019, $30,325601$.

(17) Jain, T.; Lara-Avila, S.; Kervennic, Y. V.; Moth-Poulsen, K.; Nørgaard, K.; Kubatkin, S.; Bjørnholm, T. Aligned growth of gold nanorods in PMMA channels: Parallel preparation of nanogaps. ACS Nano 2012, 6, 3861-3867.

(18) Diaz Fernandez, Y. A.; Gschneidtner, T. A.; Wadell, C.; Fornander, L. H.; Lara Avila, S.; Langhammer, C.; Westerlund, F.; Moth-Poulsen, K. The conquest of middle-earth: combining topdown and bottom-up nanofabrication for constructing nanoparticle based devices. Nanoscale 2014, 6, 14605-14616.

(19) Zhang, H.; Kinnear, C.; Mulvaney, P. Fabrication of SingleNanocrystal Arrays. Adv. Mater. 2019, 1904551. 
(20) Sayin, M.; Dahint, R. Formation of charge-nanopatterned templates with flexible geometry via layer by layer deposition of polyelectrolytes for directed self-assembly of gold nanoparticles. Nanotechnology 2017, 28, 135303.

(21) Fang, L.; Liu, X.; Xiang, S.; Liu, W.; Shen, H.; Li, Z.; Zhang, K.; Song, W.; Yang, B. Large-scale Au nanoparticle cluster arrays with tunable particle numbers evolved from colloidal lithography. Nanotechnology 2018, 29, 405301.

(22) Eklöf, J.; Gschneidtner, T.; Lara-Avila, S.; Nygård, K.; MothPoulsen, $\mathrm{K}$. Controlling deposition of nanoparticles by tuning surface charge of $\mathrm{SiO}_{2}$ by surface modifications. RSC Adv. 2016, 6, 104246104253.

(23) Eklöf-Österberg, J.; Gschneidtner, T.; Tebikachew, B.; LaraAvila, S.; Moth-Poulsen, K. Parallel Fabrication of Self-Assembled Nanogaps for Molecular Electronic Devices. Small 2018, 14, 1803471.

(24) Eklöf, J.; Stolaá, A.; Herzberg, M.; Pekkari, A.; Tebikachew, B.; Gschneidtner, T.; Lara-Avila, S.; Hassenkam, T.; Moth-Poulsen, K. Guided selective deposition of nanoparticles by tuning of the surface potential. EPL (Europhys. Lett.) 2017, 119, 18004.

(25) Derjaguin, B. Untersuchungen über die Reibung und Adhäsion, IV. Kolloid-Z. 1934, 69, 155-164.

(26) White, L. R. On the deryaguin approximation for the interaction of macrobodies. J. Colloid Interface Sci. 1983, 95, 286-288.

(27) Bhattacharjee, S.; Elimelech, M. Surface Element Integration: A Novel Technique for Evaluation of DLVO Interaction between a Particle and a Flat Plate. J. Colloid Interface Sci. 1997, 193, 273-285.

(28) Bhattacharjee, S.; Elimelech, M.; Borkovec, M. DLVO Interaction between Colloidal Particles: Beyond Derjaguin's Approximation. Croat. Chem. Acta 1998, 71, 883-903.

(29) Duffadar, R. D.; Davis, J. M. Interaction of micrometer-scale particles with nanotextured surfaces in shear flow. J. Colloid Interface Sci. 2007, 308, 20-29.

(30) Bendersky, M.; Davis, J. M. DLVO interaction of colloidal particles with topographically and chemically heterogeneous surfaces. J. Colloid Interface Sci. 2011, 353, 87-97.

(31) Shen, C.; Lazouskaya, V.; Zhang, H.; Li, B.; Jin, Y.; Huang, Y. Influence of surface chemical heterogeneity on attachment and detachment of microparticles. Colloids Surf., A 2013, 433, 14-29.

(32) Ma, H.; Pazmino, E.; Johnson, W. P. Surface Heterogeneity on Hemispheres-in-Cell Model Yields All Experimentally-Observed NonStraining Colloid Retention Mechanisms in Porous Media in the Presence of Energy Barriers. Langmuir 2011, 27, 14982-14994.

(33) Wang, Z.; Jin, Y.; Shen, C.; Li, T.; Huang, Y.; Li, B. Spontaneous Detachment of Colloids from Primary Energy Minima by Brownian Diffusion. PLoS One 2016, 11, No. e0147368.

(34) Shen, C.; Bradford, S.; Wang, Z.; Huang, Y.; Zhang, Y.; Li, B. DLVO Interaction Energies between Hollow Spherical Particles and Collector Surfaces. Langmuir 2017, 33, 10455-10467.

(35) Adamczyk, Z.; Weroński, P. Application of the DLVO theory for particle deposition problems. Adv. Colloid Interface Sci. 1999, 83, $137-226$.

(36) Hamaker, H. C. The London-van der Waals attraction between spherical particles. Physica 1937, 4, 1058-1072.

(37) Warszyński, P.; Adamczyk, Z. Calculations of Double-Layer Electrostatic Interactions for the Sphere/Plane Geometry. J. Colloid Interface Sci. 1997, 187, 283-295.

(38) Flory, P. J.; Krigbaum, W. R. Statistical Mechanics of Dilute Polymer Solutions. II. J. Chem. Phys. 1950, 18, 1086-1094.

(39) Lin, S.; Wiesner, M. R. Deposition of Aggregated Nanoparticles - A Theoretical and Experimental Study on the Effect of Aggregation State on the Affinity between Nanoparticles and a Collector Surface. Environ. Sci. Technol. 2012, 46, 13270-13277.

(40) Park, J.-W.; Shumaker-Parry, J. S. Structural Study of Citrate Layers on Gold Nanoparticles: Role of Intermolecular Interactions in Stabilizing Nanoparticles. J. Am. Chem. Soc. 2014, 136, 1907-1921.

(41) Monti, S.; Barcaro, G.; Sementa, L.; Carravetta, V.; Ågren, H. Characterization of the adsorption dynamics of trisodium citrate on gold in water solution. RSC Adv. 2017, 7, 49655-49663.
(42) Al-Johani, H.; Abou-Hamad, E.; Jedidi, A.; Widdifield, C. M.; Viger-Gravel, J.; Sangaru, S. S.; Gajan, D.; Anjum, D. H.; Ould-Chikh, S.; Hedhili, M. N.; et al. The structure and binding mode of citrate in the stabilization of gold nanoparticles. Nat. Chem. 2017, 9, 890-895.

(43) Xu, J. Q.; Chen, L. Y.; Choi, H.; Li, X. C. Theoretical study and pathways for nanoparticle capture during solidification of metal melt. J. Phys.: Condens. Matter 2012, 24, 255304.

(44) Bergström, L. Hamaker constants of inorganic materials. Adv. Colloid Interface Sci. 1997, 70, 125-169.

(45) Matsumoto, M.; Gaonkar, A. G.; Takenaka, T. The Estimation of Hamaker Constants of Alcohols and Interfacial Tensions at Alcohol-Mercury Interfaces. Bull. Inst. Chem. Res., Kyoto Univ. 1981, 58, 523-533.

(46) Ringe, S.; Oberhofer, H.; Hille, C.; Matera, S.; Reuter, K. Function-Space-Based Solution Scheme for the Size-Modified Poisson-Boltzmann Equation in Full-Potential DFT. J. Chem. Theory Comput. 2016, 12, 4052-4066.

(47) Mathew, K.; Hennig, R. G. Implicit self-consistent description of electrolyte in plane-wave density-functional theory. 2016, arXiv:1601.03346 [cond-mat]. https://arxiv.org/abs/1601.03346.

(48) Myint, M.; Yan, Y.; Chen, J. G. Reaction Pathways of Propanal and 1-Propanol on $\mathrm{Fe} / \mathrm{Ni}(111)$ and $\mathrm{Cu} / \mathrm{Ni}(111)$ Bimetallic Surfaces. J. Phys. Chem. C 2014, 118, 11340-11349.

(49) Mizukami, M.; Kurihara, K. Hydrogen-Bonded Macrocluster Formation of 1-Propanol and 2-Propanol on Silica Surfaces. Aust. J. Chem. 2003, 56, 1071-1080.

(50) Takeuchi, Y.; Ida, T.; Kimura, K. Colloidal Stability of Gold Nanoparticles in 2-Propanol under Laser Irradiation. J. Phys. Chem. B 1997, 101, 1322-1327.

(51) Martell, A. E.; Hancock, R. D. Metal Complexes in Aqueous Solutions; Modern Inorganic Chemistry; Springer: US, 1996.

(52) Shinohara, S.; Eom, N.; Teh, E.-J.; Tamada, K.; Parsons, D.; Craig, V. S. J. The Role of Citric Acid in the Stabilization of Nanoparticles and Colloidal Particles in the Environment: Measurement of Surface Forces between Hafnium Oxide Surfaces in the Presence of Citric Acid. Langmuir 2018, 34, 2595-2605. 